\title{
ON DIFFERENTIALS OF THE FIRST KIND AND THETA CONSTANTS FOR CERTAIN CONGRUENCE SUBGROUPS
}

BY

\section{A. J. CRISALLI}

ABSTRACT. Let $\Gamma(8)$ denote the principal congruence subgroup of level 8 and let $\Gamma(16,32)$ denote the subgroup of $\Gamma(16)$ satisfying $c d \equiv$ $a b \equiv 0(\bmod 32)$. We are dealing only with the elliptic modular case. Consider the spaces of cusp forms of weight 2 (differentials of the first kind) with respect to these groups. It is proved that these spaces are generated by certain monomials of theta constants of degree 4 .

Introduction. Let $\Gamma(1)$ denote the group $S L(2, \mathrm{Z})$ and let $\Gamma(n)$ denote the principal congruence subgroup of $\Gamma(1)$ of level $n$. If $r$ is an even positive integer let $\Gamma(r, 2 r)$ denote the subgroup of $\Gamma(r)$ defined in [3, p. 222]. If

$$
A\left(\Gamma\left(r^{2}, 2 r^{2}\right)\right)=\bigoplus \sum_{k \geq 0} A\left(\Gamma\left(r^{2}, 2 r^{2}\right)\right)_{k}
$$

denotes the graded ring of modular forms for the group $\Gamma\left(r^{2}, 2 r^{2}\right)$, the $k$ th component being defined by $f(M(z))=(c z+d)^{k} f(z)$ and certain holomorphicity conditions, and if $\mathscr{Q}(r)=\bigoplus \Sigma_{k \geq 0} \mathbb{Q}(r)_{k}$ denotes the graded ring whose $k$ th component is the vector space generated by monomials of theta constants $\Pi_{a=1}^{2 k} \theta\left[m_{\alpha}\right]$ such that $r m_{\alpha} \equiv 0(\bmod 1)$, where theta constants are defined in $\S 2$, then by $[3$, p. 235] the integral closure of $\mathscr{Q}(r)$ in its field of fractions is $A\left(\Gamma\left(r^{2}, 2 r^{2}\right)\right)$. When $r=2, \mathfrak{Q}(2)=A(\Gamma(4,8))$ but it is known ([1] or [4, p. 244]) that $\mathfrak{Q}(4)_{2}$ is properly contained in $A(\Gamma(16,32))_{2}$. However the possibility exists that the cusp forms in $\mathscr{Q}(r)_{2}$ generate the entire space of cusp forms in $A\left(\Gamma\left(r^{2}, 2 r^{2}\right)\right)_{2}$. The purpose of this paper is to show that the cusp forms in $\mathfrak{Q}(4){ }_{2}$ generate the entire space of cusp forms in $A(\Gamma(16,32))_{2}$ and that a similar fact holds for the group $\Gamma(8)$. We shall also obtain explicit bases for these spaces in terms of theta constants.

Received by the editors April 2, 1974.

AMS (MOS) subject classifications (1970). Primary 10D05; Secondary 10D20.

Key words and phrases. Congruence subgroup, cusp form of weight 2, differential of the first kind, theta constant. 
The groups $\Gamma(2), \Gamma(4), \Gamma(4,8)$ were treated in [1], where similar results were obtained. The basic technique we will use is the characteristic based direct sum decomposition of vector spaces of theta constants. The cusp forms of weight 2 for any of the above groups induce the differentials of the first kind on the ir associated Riemann surfaces, hence the title of this paper.

1. Determination of an orbit. If $x$ and $y$ are in $\mathbf{R}^{2}$ define $x \sim y$ to mean $x \equiv y(\bmod 1)$ or $x \equiv-y(\bmod 1)$. This is an equivalence relation. For a given pair $(M, m), M$ an element of $\Gamma(1)$ and $m=\left(m^{\prime}, m^{\prime \prime}\right)$ an element of $\mathbf{R}^{2}$, define $M m=\left(d m^{\prime}-c m^{\prime \prime},-b m^{\prime}+a m^{\prime \prime}\right)+1 / 2(c d, a b)$. Throughout it will be understood that $M=\left(\begin{array}{ll}a & b \\ c & d\end{array}\right)$. Unless otherwise specified it is also understood that $M m$ is not the matrix product of $M$ and $m$.

Clearly, $x \sim y$ implies $M x \sim M y$, and $M_{1}, M_{2}$ in $\Gamma(1)$ implies $\left(M_{1} M_{2}\right) x$ $\sim M_{1}\left(M_{2} x\right)$. In fact, by [3, p. 222], $\left(M_{1} M_{2}\right) x \equiv M_{1}\left(M_{2} x\right)(\bmod 1)$. Also $1_{2} x \sim x$. The se facts mean that the group $\Gamma(1)$ operates on the set of $\sim$ classes in $\mathbf{R}^{2}$. If $r$ is an even positive integer let $Q(r)$ denote the set of all $(i / r, j / r)$, $i, j$ in $\mathbf{Z}$. If $\overline{Q(r)}$ denotes the set of $\sim$ classes in $Q(r)$ then $\Gamma(1)$ operates on the set $\overline{Q(r)}$ by restriction. A system of representatives for the $\sim$ classes in $Q(r)$ is given by the set $S_{r}$ consisting of all $(i / r, j / r)$ such that $0<i / r<1 / 2,0 \leq j / r<1$, or $i / r=1 / 2,0 \leq j / r<1 / 2$, or $i / r=0,0 \leq j / r \leq 1 / 2$.

Let $e=\left(\begin{array}{ll}0 & 0\end{array}\right), f=\left(\begin{array}{ll}01 / 2\end{array}\right), g=\left(\begin{array}{ll}0 & 1 / 4\end{array}\right)$ be elements of $\mathbf{R}^{2}$. The subset of $\Gamma(1)$ consisting of all $M$ such that $M e \sim e, M f \sim f, M g \sim g$ is clearly a subgroup which will be denoted by $G(e, f, g)$.

Lemma 1. $G(e, f, g)$ is the subgroup of $\Gamma(2)$ consisting of those $M$ such that $c \equiv 0(\bmod 4)$.

Proof. If $M$ satisfies the stated conditions then $b \equiv 0(\bmod 2)$ and $c \equiv 0(\bmod 2)$, which implies that, for any $m$ in $\mathbf{R}^{2}, M m \equiv\left(d m^{\prime}-c m^{\prime \prime}\right.$, $\left.-b m^{\prime}+a m^{\prime \prime}\right)(\bmod 1)$. Hence $M e \equiv e(\bmod 1)$ and $M f \equiv(-c / 2, a / 2)(\bmod 1)$. Since $c \equiv 0(\bmod 4), a \equiv 1(\bmod 2)$ we have $M f \equiv f(\bmod 1)$. Also, $M g \equiv$ $(-c / 4, a / 4) \equiv(0, a / 4)(\bmod 1)$ and $(0, a / 4) \sim g$ the latter because $a \equiv$ $1(\bmod 2)$. Thus $M$ is in $G(e, f, g)$. Conversely, if $M$ is in $G(e, f, g)$ and $m$ is $e, f$, or $g$ we have $\left(d m^{\prime}-c m^{\prime \prime}+1 / 2 c d,-b m^{\prime}+a m^{\prime \prime}+1 / 2 a b\right) \equiv \pm m(\bmod 1)$. Letting $m=e$ implies $c d \equiv 0(\bmod 2)$ and $a b \equiv 0(\bmod 2)$. Letting $m=f$ implies $c d \equiv c(\bmod 2)$ and $a+a b \equiv 1(\bmod 2)$. Thus $c \equiv 0(\bmod 2)$ and $a \equiv 1(\bmod 2)$. The latter along with $a b \equiv 0(\bmod 2)$ implies that $b \equiv 0(\bmod 2)$. Since $a d-b c=1, b c$ even, we have $d \equiv 1(\bmod 2)$. Letting $m=g$ yields $-c / 4+c d / 2 \equiv 0(\bmod 1)$. Thus $c(2 d-1) \equiv 0(\bmod 4)$ which implies $c \equiv$ $0(\bmod 4)$. 
Lemma 2. The group index of $G(e, f, g)$ in $\Gamma(1)$ is 12.

Proof. By Lemma $1, G(e, f, g)$ is contained in $\Gamma(2)$ and the inclusion is proper by observing the matrix $M=\left(\begin{array}{ll}3 & 4 \\ 2 & 3\end{array}\right)$. The group $\Gamma(2,4)$ is contained in $G(e, f, g)$ because if $M$ is in $\Gamma(2,4)$ then $M$ is in $\Gamma(2)$ and $c d \equiv 0(\bmod 4)$, $d \equiv 1(\bmod 2)$, implying that $c \equiv 0(\bmod 4)$. The inclusion is proper as can be seen from the matrix $M=\left(\begin{array}{cc}1 & 2 \\ -4 & -7\end{array}\right)$. These facts imply that $[\Gamma(2)$ : $G(e, f, g)]$ is a proper divisor of $[\Gamma(2): \Gamma(2,4)]$. Since the latter is 4 by $[2$, p. 222] we see that $[\Gamma(2): G(e, f, g)]=2$. Thus the required index is $[\Gamma(1): \Gamma(2)][\Gamma(2): G(e, f, g)]=6 \cdot 2=12$.

Theorem 1. Let $e=\left(\begin{array}{ll}0 & 0\end{array}\right), f=\left(\begin{array}{ll}0 & 1 / 2\end{array}\right), g=\left(\begin{array}{ll}0 & 1 / 4\end{array}\right), M_{1}=\left(\begin{array}{ll}1 & 0 \\ 1 & 1\end{array}\right), M_{2}=$ $\left(\begin{array}{cc}-1 & -1 \\ 3 & 2\end{array}\right), M_{3}=\left(\begin{array}{ll}1 & 0 \\ 2 & 1\end{array}\right)$. Then a system of representatives for the cosets $g G(e, f, g), g \in \Gamma(1)$, is given by $1_{2}, M_{1}, M_{2}, M_{2}^{2}, M_{1} M_{2}, M_{2} M_{1}, M_{3}, M_{1} M_{3}$, $M_{2} M_{3}, M_{2}^{2} M_{3}, M_{1} M_{2} M_{3}, M_{2} M_{1} M_{3}$. The values taken by $(M e, M f, M g) \bmod \sim$ as $M$ runs through the above representatives in the order stated are

$$
\begin{aligned}
& \left(\begin{array}{llllll}
0 & 0,0 & 1 / 2,0 & 1 / 4
\end{array}\right), \quad\left(1 / 20,01 / 2,1 / 4 \frac{1}{4}\right), \quad\left(0 \frac{1}{2}, 1 / 20,1 / 4 \frac{1}{4}\right) \text {, } \\
& (1 / 20,00,1 / 41 / 2), \quad(01 / 2,00,1 / 21 / 4), \quad\left(00,1 / 20,1 / 4 \frac{1}{2}\right) \text {, } \\
& \left(0 \quad 0,0 \frac{1}{2}, 1 / 2 \frac{1}{4}\right), \quad\left(1 / 20,0 \frac{1}{2}, 1 / 43 / 4\right), \quad\left(0 \frac{1}{2}, 1 / 20,1 / 4 \frac{3}{4}\right) \text {, } \\
& (1 / 20,00,1 / 40), \quad(01 / 2,00,01 / 4), \quad(00,1 / 20,1 / 40) \text {, }
\end{aligned}
$$

and these are all possible values of $(\mathrm{Me}, \mathrm{Mf}, \mathrm{Mg}) \bmod \sim$ as $M$ runs through all elements of $\Gamma(1)$.

Proof. Direct computation shows that as $M$ runs through the above stated system, ( $M e, M f, M g$ ) runs through the above stated values mod $\sim$ Since there are 12 of them, the theorem now follows from Lemma 2.

2. Monomial cusp forms. In the following $\tau$ denotes a point in the upper half plane, $e(z)=e^{2 \pi i z}$ and $m=\left(m^{\prime}, m^{\prime \prime}\right)$ is in $\mathbf{R}^{2}$. The holomorphic function in $r$ defined by the series

$$
\theta[m](\tau)=\sum_{p \in Z} e\left[\left(p+m^{\prime}\right) m^{\prime \prime}+1 / 2\left(p+m^{\prime}\right)^{2} \tau\right]
$$

is called the theta constant of characteristic $m$. Sume elementary properties of theta constants are $\theta[m](\tau)=\theta[-m](\tau), \theta[m+n](\tau)=e\left(m^{\prime} n^{\prime \prime}\right) \theta[m](\tau)$ where $n$ is any element of $\mathrm{Z}^{2}$.

If $m$ is in $\mathbf{Q}^{2}, \theta[m](\tau)$ vanishes identically in $r$ if and only if $m \equiv$ $(1 / 21 / 2)(\bmod 1)$. Hence if $m \sim s$ then $\theta[m]$ is a scalar multiple of $\theta[s]$ and every nonzero $\theta[m]$ such that $r m \equiv 0(\bmod 1)$ is a scalar multiple of some $\theta[s], s \in s_{r}$ 
If $f(\tau)$ is a holomorphic function defined in the upper half plane, $M$ is in $\Gamma(1)$, and $k \geq 0$ is an integer, define $f_{k} M=f(M(\tau))(c \tau+d)^{-k}$, where $M(\tau)$ is the linear fractional transformation induced by $M$. The monomials of theta constants of degree $2 k$ satisfy the following transformation law [3, p. 228]:

$$
\left.\left(\prod_{a=1}^{2 k} \theta\left[m_{a}\right]\right)\right|_{k} M=\chi(M)^{2 k} e\left(\sum_{a=1}^{2 k} \phi\left[M^{-1} m_{a}\right](M)\right) \prod_{a=1}^{2 k} \theta\left[M^{-1} m_{a}\right]
$$

where $\phi[m](M)=-1 / 2\left(m^{\prime 2} b d+m^{\prime \prime 2} a c-2 m^{\prime} m^{\prime \prime} b c-a b\left(d m^{\prime}-c m^{\prime \prime}\right)\right)$ and $\chi(M)$ denotes a certain scalar depending only on $M$. A lengthy computation using $a d-b c=1$ yields

$$
\begin{aligned}
\phi\left[M^{-1} m_{a}\right]=-1 / 2( & m^{\prime 2} b a+m^{\prime \prime 2} c d+2 m^{\prime} m^{\prime \prime} b c \\
& \left.+m^{\prime}(a b)(-a c d-b c d-1)+m^{\prime \prime}(a b c d)(-c-d)+C(M)\right)
\end{aligned}
$$

where $C(M)$ is a scalar depending only on $M$, not on $m_{a}$. The transformation law is then

$$
\begin{aligned}
& \left.\prod_{a=1}^{2 k} \theta\left[m_{a}\right]\right|_{k} M \\
& =L(M) e\left[-1 / 2\left(b a \sum_{a=1}^{2 k} m_{a}^{\prime 2}+c d \sum_{a=1}^{2 k} m_{a}^{\prime \prime 2}+2 b c \sum_{a=1}^{2 k} m_{a}^{\prime} m_{a}^{\prime \prime}\right.\right. \\
& \left.\left.\quad+a b(-a c d-b c d-1) \sum_{a=1}^{2 k} m_{a}^{\prime}+(a b c d)(-c-d) \sum_{a=1}^{2 k} m_{a}^{\prime \prime}\right)\right],
\end{aligned}
$$

where $L(M)$ is a scalar depending only on $M$.

Theorem 2. Let $\Pi_{a=1}^{2 k} \theta\left[m_{a}\right]$ be in $\mathbb{Q}(r)_{k}$. Then $\Pi_{a=1}^{2 k} \theta\left[m_{a}\right]$ is not a cusp form of weight $k$ for $\Gamma\left(r^{2}, 2 r^{2}\right)$ if and only if there exist $M$ in $\Gamma(1)$ and $s_{a}=\left(s_{a}^{\prime}, s_{a}^{\prime \prime}\right)$ in $\mathbf{R}^{2}, s_{\alpha}^{\prime} \equiv 0(\bmod 1), \alpha=1, \ldots, 2 k$, such that $M s_{a}=m_{a^{\prime}}$ $a=1, \ldots, 2 k$.

Proof. Let $f=\Pi_{a=1}^{2 k} \theta\left[m_{a}\right] . f$ is a cusp form of weight $k$ for $\Gamma\left(r^{2}, 2 r^{2}\right)$ if and only if, for every $M$ in $\Gamma(1),\left.f\right|_{k} M$ has 0 th Fourier coefficient with respect to $\Gamma\left(r^{2}, 2 r^{2}\right)$ equal to 0 . By the transformation formula this is equivalent to saying that $\Pi_{a=1}^{2 k} \theta\left[M^{-1} m_{a}\right]$ has vanishing 0 th Fourier coefficient for all $M$ in $\Gamma(1)$. This means that, for some $a, M^{-1} m_{\alpha}=\left(s_{\alpha}^{\prime}, s_{\alpha}^{\prime \prime}\right)$, 
$s_{a}^{\prime} \not \equiv 0(\bmod 1)$, as can be seen from the Fourier expansion

$$
\prod_{a=1}^{2 k} \theta\left[m_{a}\right]=\sum_{\lambda \geq 0} a_{\lambda} e\left(\lambda \tau / 2 r^{2}\right), \quad a_{\lambda}=\sum e\left(\sum_{a=1}^{2 k} x_{a} m_{a}^{\prime \prime} / r\right),
$$

the summation running through all $\left(x_{1}, \ldots, x_{2 k}\right)$ in $\mathrm{Z}^{2 k}$ satisfying $\Sigma_{a=1}^{2 k} x_{a}^{2}=\lambda, x_{a} \equiv r m_{a}^{\prime}(\bmod r), \alpha=1, \ldots, 2 k$.

Corollary. Consider the collection of monomials of theta constants in $\mathfrak{Q}(4)_{k}$ of type $\Pi_{a=1}^{2 k} \theta\left[m_{a}\right]$ where $m_{a} \in S_{4}, \alpha=1, \ldots, 2 k$. The monomials in the above collection which are not cusp forms for $\Gamma(16,32)$ are those of the following type in which $n_{1}, n_{2}, n_{3} \geq 0, n_{1}+n_{2}+n_{3}=2 k$ :

$$
\begin{aligned}
& \theta\left[\begin{array}{ll}
0 & 0
\end{array}\right]^{n} 1 \theta\left[\begin{array}{ll}
0 & 1 / 2
\end{array}\right]^{n} 2 \theta\left[\begin{array}{ll}
0 & 1 / 4
\end{array}\right]^{n}, \quad \theta\left[\begin{array}{ll}
1 / 2 & 0
\end{array}\right]^{n} 1 \theta\left[\begin{array}{ll}
0 & 1 / 2
\end{array}\right]^{n} 2 \theta\left[\begin{array}{ll}
1 / 4 & 1 / 4
\end{array}\right]^{n}, \\
& \theta\left[\begin{array}{ll}
1 / 2 & 0
\end{array}\right]^{n} \theta\left[\begin{array}{ll}
0 & 0
\end{array}\right]^{n} 2 \theta\left[\begin{array}{ll}
1 / 4 & 1 / 2
\end{array}\right]^{n}, \quad \theta\left[\begin{array}{ll}
0 & 1 / 2
\end{array}\right]^{n} \theta\left[\begin{array}{ll}
0 & 0
\end{array}\right]^{n} 2 \theta\left[\begin{array}{ll}
1 / 2 & 1 / 4
\end{array}\right]^{n}, \\
& \theta\left[\begin{array}{ll}
1 / 2 & 0
\end{array}\right]^{n} \theta\left[\begin{array}{ll}
0 & 1 / 2
\end{array}\right]^{n} \theta\left[\begin{array}{lll}
1 / 4 & 3 / 4
\end{array}\right]^{n}, \quad \theta\left[\begin{array}{ll}
1 / 2 & 0
\end{array}\right]^{n} \theta\left[\begin{array}{ll}
0 & 0
\end{array}\right]^{n} 2 \theta\left[\begin{array}{ll}
1 / 4 & 0
\end{array}\right]^{n} 3 .
\end{aligned}
$$

A monomial in $\mathscr{Q}(4)_{k}$ is not a cusp form if and only if it is a scalar multiple of one of these.

Proof. The Corollary follows from Theorems 1 and 2, and the elementary facts about theta constants stated at the beginning of this section.

We shall count the total number $T$ of monomials of degree $2 k$ in the above Corollary. There are 6 types. Let $T_{i}, 1 \leq i \leq 6$, denote the number of monomials in the $i$ th type such that $n_{3}>0$ and let $S$ denote the total number of monomials of degree $2 k$ involving at most 2 of $\left(\begin{array}{ll}0 & 0\end{array}\right),\left(\begin{array}{ll}0 & 1 / 2\end{array}\right)$, $(1 / 20)$. Then $T=\sum_{i=1}^{6} T_{i}+S$. If $N(d, n)$ denotes the total number of monomials of degree $d$ in $n$ variables then $T_{i}=N(2 k-1,3)$ and $S=N(2 k, 2)+$ $2 N(2 k-1,2)-1$. Thus $T=12 k(k+1)$. The total number of monomials $\Pi_{a=1}^{2 k} \theta\left[m_{a}\right], m_{a} \in S_{4}$, is $N(2 k, 9)$ because there are 9 elements in $S_{4}$. If $N_{1}=$ number of monomials $\Pi_{a=1}^{4} \theta\left[m_{a}\right], m_{a} \in S_{4}, N_{2}=$ number of monomial cusp forms of this type, and $N_{3}=$ number of monomial noncusp forms of this type then $N_{1}=495, N_{2}=423, N_{3}=72$.

3. Another type of cusp form. In the preceding section we determined all monomial cusp forms in $\mathbb{Q}(4)_{k}$. We shall now find some cusp forms not of this form.

Lemma 3. For each $M$ in $\Gamma(1)$ we have $M\left(\begin{array}{lll}0 & 1 / 4\end{array}\right) \sim\left(\begin{array}{ll}0 & 1 / 4\end{array}\right)$ if and only if $c \equiv 0(\bmod 4)$. 
Proof. If $M\left(\begin{array}{ll}0 & 1 / 4\end{array}\right) \sim\left(\begin{array}{ll}0 & 1 / 4\end{array}\right)$ then $(-c / 4+c d / 2, a / 4+a b / 2) \equiv(0, \pm 1 / 4)$ $(\bmod 1)$ and so $c(2 d-1) \equiv 0(\bmod 4)$. Thus $c \equiv 0(\bmod 4)$. Conversely, if $c \equiv 0(\bmod 4)$ then $M(01 / 4)=(-c / 4+c d / 2, a / 4+a b / 2) \equiv(0, a / 4+a b / 2) \sim$ ( $01 / 4$ ) because $a d-b c=1$ implies $a$ is odd.

Lemma 4. If $m$ is in $\mathbf{R}^{2}, m \sim\left(\begin{array}{ll}0 & x\end{array}\right)$ then $\theta[m]=\theta[0 x]$.

Proof. This follows immediately from the 2 identities stated at the beginning of $\S 2$.

Theorem 3. $\theta\left[\begin{array}{ll}0 & 1 / 4\end{array}\right]\left[\begin{array}{ll}0 & 0\end{array}\right]^{3}-\theta\left[\begin{array}{ll}0 & 1 / 4\end{array}\right]\left[\begin{array}{ll}0 & 1 / 2\end{array}\right]^{2} \theta\left[\begin{array}{ll}0 & 0\end{array}\right]$ is a cusp form of weight 2 for the group $\Gamma(16,32)$.

Proof. Let $/$ be the stated expression. It suffices to show that, for every $M$ in $\Gamma(1),\left.f\right|_{k} M$ has vanishing 0 th Fourier coefficient. We divide the proof into two cases. In the first case suppose that $M^{-1}\left(\begin{array}{ll}0 & 1 / 4\end{array}\right)=\left(\begin{array}{ll}a^{\prime} & a^{\prime \prime}\end{array}\right)$ where $a^{\prime} \not \equiv 0(\bmod 1)$. The transformation law gives $\left.f\right|_{k} M=a$ linear combi-

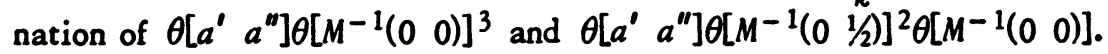
Since $a^{\prime} \equiv 0(\bmod 1)$ a look at the definition of $\theta[\mathrm{m}]$ shows that the 0 th Fourier coefficient of $\theta\left[a^{\prime} a^{\prime \prime}\right]$ is 0 . Thus the same is true of $\left.f\right|_{k} M$. In the

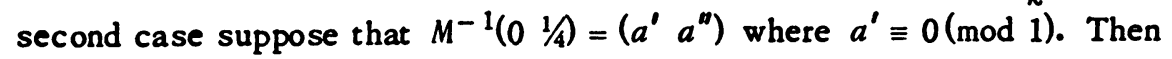
$M^{-1}\left(\begin{array}{ll}0^{1 / 4}\end{array}\right) \sim\left(\begin{array}{ll}0^{1 / 4}\end{array}\right)$ as can be seen from Theorem 1 . Thus $\left(\begin{array}{ll}0 & 1 / 4\end{array}\right) \sim M\left(\begin{array}{ll}0 & 1 / 4\end{array}\right)$ and Lemma 3 implies $c \equiv 0(\bmod 4)$. The table in Theorem 1 actually shows that the triple $\left(M^{-1}(00), M^{-1}\left(\begin{array}{ll}0 & 1\end{array} / 2\right), M^{-1}\left(\begin{array}{ll}0 & 1 / 4\end{array}\right)\right)$ is $\sim$ to either $\left(\left(\begin{array}{ll}0 & 0\end{array}\right)\right.$, $\left.\left(\begin{array}{ll}0 & 1 / 2\end{array}\right),\left(\begin{array}{ll}1 / 4\end{array}\right)\right)$ or to $\left(\left(\begin{array}{ll}0 & 1 / 2\end{array}\right),\left(\begin{array}{ll}0 & 0\end{array}\right),\left(\begin{array}{ll}0 & 1 / 4\end{array}\right)\right.$. Lemma 4 then implies that

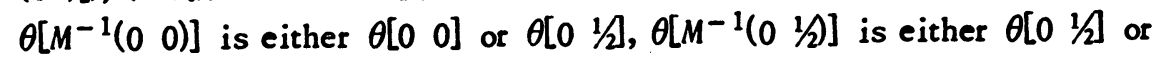
$\theta\left[\begin{array}{ll}0 & 0\end{array}\right]$ and $\theta\left[M^{-1}\left(\begin{array}{ll}0 & 1 / 4\end{array}\right)\right]$ is $\theta\left[\begin{array}{ll}0 & 1 / 4\end{array}\right]$. Hence they all have 0 th Fourier coefficient equal to 1 . The transformation law yields

$$
\begin{aligned}
f I_{k} M= & C_{1}(M) \theta\left[M ^ { - 1 } ( \begin{array} { l l } 
{ 0 } & { 1 / 4 }
\end{array} ] \theta \left[M^{-1}\left(\begin{array}{ll}
0 & 0
\end{array}\right]^{3}\right.\right. \\
& -C_{2}(M) \theta\left[M ^ { - 1 } ( \begin{array} { l l } 
{ 0 } & { 1 / 4 }
\end{array} ] \theta \left[M ^ { - 1 } ( \begin{array} { l l } 
{ 0 } & { 1 / 2 }
\end{array} ] ^ { 2 } \theta \left[M^{-1}\left(\begin{array}{ll}
0 & 0
\end{array}\right]\right.\right.\right.
\end{aligned}
$$

where $C_{1}(M)$ and $C_{2}(M)$ are scalars depending only on $M$. The 0th Fourier coefficient of $\left.f\right|_{k} M$ is thus $C_{1}(M)-C_{2}(M)$, and we have only to show it vanishes. From the version of the transformation formula we found in $\$ 2$, we have, setting all the $m_{\alpha}^{\prime}=0$,

$$
\begin{aligned}
& \left.C_{1}(M)=L(M) e(-1 / 2(1 / 1 G) c d+(1 / 4)(a b c d)(-c-d))\right), \\
& C_{2}(M)=L(M) e(-1 / 2((1 / 16+1 / 4+1 / 4) c d+(1 / 4+1 / 2+1 / 2)(a b c d)(-c-d))) .
\end{aligned}
$$


So $C_{2}(M) / C_{1}(M)=e(-1 / 2(1 / 2 c d+a b c d(-c-d)))=1$, the last equality following from $c \equiv 0(\bmod 4)$. This proves case 2 and hence the entire theorem.

Corollary. Let

$$
C_{1}(M)=e\left(\phi\left[M\left(\begin{array}{ll}
0 & 1 / 4
\end{array}\right)\right]\left(M^{-1}\right)+3 \phi[M(0 \quad 0)]\left(M^{-1}\right)\right)
$$

and

$$
C_{2}(M)=e\left(\phi\left[M\left(0^{1 / 4}\right)\right]\left(M^{-1}\right)+2 \phi\left[M\left(0^{1 / 2}\right)\right]\left(M^{-1}\right)+\phi\left[M \left(\begin{array}{ll}
0 & \left.0)]\left(M^{-1}\right)\right)
\end{array}\right.\right.\right.
$$

The following are cusp forms for $\Gamma(16,32)$ where $M_{1}, M_{2}, M_{3}$ are defined in Theorem 1:

$\theta\left[\begin{array}{ll}0 & 0\end{array}\right]^{3} \theta\left[\begin{array}{ll}0 & 1 / 4\end{array}\right]-\theta\left[\begin{array}{ll}0 & 0\end{array}\right] \theta\left[\begin{array}{ll}0 & 1 / 2\end{array}\right]^{2} \theta\left[\begin{array}{ll}0 & 1 / 4\end{array}\right]$,

$C_{1}\left(M_{1}\right) \theta[1 / 20]^{3} \theta\left[\begin{array}{ll}1 / 4 & 1 / 4\end{array}\right]-C_{2}\left(M_{1}\right) \theta[1 / 20] \theta\left[\begin{array}{ll}0 & 1 / 2\end{array}\right]^{2} \theta\left[\begin{array}{ll}1 / 4 & 1 / 4\end{array}\right]$

$C_{1}\left(M_{2}\right) \theta\left[\begin{array}{ll}0 & 1 / 2\end{array}\right]^{3} \theta\left[\begin{array}{ll}1 / 4 & 1 / 4\end{array}\right]-C_{2}\left(M_{2}\right) \theta\left[\begin{array}{ll}0 & 1 / 2\end{array}\right] \theta\left[\begin{array}{ll}1 / 2 & 0\end{array}\right]^{2} \theta\left[\begin{array}{ll}1 / 4 & 1 / 4\end{array}\right]$

$C_{1}\left(M_{2}^{2}\right) \theta\left[\begin{array}{ll}1 / 2 & 0\end{array}\right]^{3} \theta\left[\begin{array}{ll}1 / 4 & 1 / 2\end{array}-C_{2}\left(M_{2}^{2}\right) \theta\left[\begin{array}{ll}1 / 2 & 0\end{array}\right] \theta\left[\begin{array}{ll}0 & 0\end{array}\right]^{2} \theta\left[\begin{array}{ll}1 / 4 & 1 / 2\end{array}\right.\right.$,

$C_{1}\left(M_{1} M_{2}\right) \theta\left[\begin{array}{ll}0 & 1 / 2\end{array}\right]^{3} \theta\left[\begin{array}{ll}1 / 2 & 1 / 4\end{array}\right]-C_{2}\left(M_{1} M_{2}\right) \theta\left[\begin{array}{ll}0 & 1 / 2\end{array}\right] \theta\left[\begin{array}{ll}0 & 0\end{array}\right]^{2} \theta\left[\begin{array}{ll}1 / 2 & 1 / 4\end{array}\right]$,

$C_{1}\left(M_{2} M_{1}\right) \theta\left[\begin{array}{ll}0 & 0\end{array}\right]^{3} \theta\left[\begin{array}{ll}1 / 4 & 1 / 2\end{array}\right]-C_{2}\left(M_{2} M_{1}\right) \theta\left[\begin{array}{ll}0 & 0\end{array}\right] \theta\left[\begin{array}{ll}0 & 1 / 2\end{array}\right]^{2} \theta\left[\begin{array}{ll}1 / 4 & 1 / 2\end{array}\right]$,

$C_{1}\left(M_{3}\right) \theta\left[\begin{array}{ll}0 & 0\end{array}\right]^{3} \theta\left[\begin{array}{ll}1 / 2 & 1 / 4\end{array}\right]-C_{2}\left(M_{3}\right) \theta\left[\begin{array}{ll}0 & 0\end{array}\right] \theta\left[\begin{array}{ll}0 & 1 / 2\end{array}\right]^{2} \theta\left[\begin{array}{ll}1 / 2 & 1 / 4\end{array}\right]$,

$C_{1}\left(M_{1} M_{3}\right) \theta\left[\begin{array}{ll}1 / 2 & 0\end{array}\right]^{3} \theta\left[\begin{array}{ll}1 / 4 & 3 / 4\end{array}\right]-C_{2}\left(M_{1} M_{3}\right) \theta[1 / 20] \theta\left[\begin{array}{ll}0 & 1 / 2\end{array}{ }^{2} \theta[1 / 43 / 4\right.$,

$C_{1}\left(M_{2} M_{3}\right) \theta\left[\begin{array}{ll}0 & 1 / 2\end{array}\right]^{3} \theta\left[1 / 43 / 4-C_{2}\left(M_{2} M_{3}\right) \theta\left[\begin{array}{ll}0 & 1 / 2\end{array}\right] \theta\left[\begin{array}{ll}1 / 2 & 0\end{array}\right]^{2} \theta[1 / 4 \quad 3 / 4]\right.$,

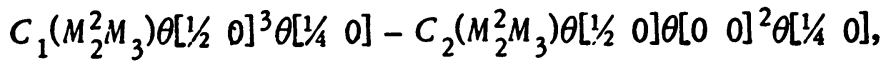

$C_{1}\left(M_{1} M_{2} M_{3}\right) \theta\left[\begin{array}{ll}0 & 1 / 2\end{array}\right]^{3} \theta\left[\begin{array}{ll}0 & 1 / 4\end{array}\right]-C_{2}\left(M_{1} M_{2} M_{3}\right) \theta\left[\begin{array}{ll}0 & 1 / 2\end{array}\right] \theta\left[\begin{array}{ll}0 & 0\end{array}\right]^{2} \theta\left[\begin{array}{ll}0 & 1 / 4\end{array}\right]$,

$C_{1}\left(M_{2} M_{1} M_{3}\right) \theta[00]^{3} \theta\left[\begin{array}{ll}1 / 4 & 0\end{array}-C_{2}\left(M_{2} M_{1} M_{3}\right) \theta\left[\begin{array}{ll}0 & 0\end{array}\right]\left[\begin{array}{ll}1 / 2 & 0\end{array}\right]^{2} \theta\left[\begin{array}{ll}1 / 4 & 0\end{array}\right]\right.$.

Proof. If $f$ is any cusp form then so is $\left.f\right|_{k} M$ for any $M$ in $\Gamma(1)$. Letting $M$ run through the inverses of the 12 matrices in Theorem 1 and using the transformation formula proves the Corollary.

4. The spaces $C(\Gamma(8))_{2}$ and $C(\Gamma(16,32))_{2}$. The spaces of cusp forms of weight 2 for the groups $\Gamma(8)$ and $\Gamma(16,32)$ are denoted by $C(\Gamma(8))_{2}$ and 
$C(\Gamma(16,32))_{2}$ respectively. We shall determine bases for these spaces in terms of theta constants. In order to accomplish this we shall develop some direct sum decompositions of the space $\mathscr{Q}(4)_{2}$.

Let $r$ be an even positive integer. Contrary to previous usage we shall throughout this section agree to view elements of $S_{r}$ as 2-component columns (rather than rows). Let $S_{r}^{2 k}$ be the $2 k$-fold Cartesian product of $S_{r}$. Thus a typical element of $S_{r}^{2 k}$ has the form

$$
\left(\begin{array}{ccc}
x_{1}^{\prime} & \cdots & x_{2 k}^{\prime} \\
x_{1}^{\prime \prime} & \cdots & x_{2 k}^{\prime \prime}
\end{array}\right) .
$$

Construct an undirected graph, denoted by $\operatorname{Graph}\left(S_{r}^{2 k}\right)$, whose points are the elements of $S_{r}^{2 k}$ and such that two points $x$ and $y$ are joined by an arc if and only if there exist $a$ and $b$ in $Z^{2 k}$ such that $a_{a} \equiv r x_{\alpha}^{\prime}(\bmod r), b_{a} \equiv$ $r y_{\alpha}^{\prime}(\bmod r), a=1, \ldots, 2 k, \Sigma_{a=1}^{2 k} a_{a}^{2}=\Sigma_{a=1}^{2 k} b_{a}^{2}$. If $x=\left(x_{1}, \ldots, x_{2 k}\right)$ is in $s_{r}^{2 k}$, define $M x=\left(M x_{1}, \ldots, M x_{2 k}\right)$.

Lemma 5. If the points $x$ and $y$ of $\operatorname{Graph}\left(S_{r}^{2 k}\right)$ are connected by an arc then

$$
\sum_{a=1}^{2 k}\left(r x_{a}^{\prime}\right)^{2} \equiv \sum_{a=1}^{2 k}\left(r y_{a}^{\prime}\right)^{2} \bmod (r \operatorname{gcd}(r, 2 d, 2 e))
$$

where $d=\operatorname{gcd}\left(r x_{a}^{\prime}\right), e=\operatorname{gcd}\left(r y_{a}^{\prime}\right)$.

Proof. Since $a_{a} \equiv r x_{a}^{\prime}(\bmod r)$,

$$
\sum a_{\alpha}^{2}-\sum\left(r x_{\alpha}^{\prime}\right)^{2}=\sum\left(a_{\alpha}-r x_{\alpha}^{\prime}\right)\left(a_{\alpha}-r x_{\alpha}^{\prime}+2 r x_{\alpha}^{\prime}\right) \equiv 0 \bmod (r \operatorname{gcd}(r, 2 d)) .
$$

Combining this with a similar congruence involving $r y_{a}^{\prime}$ yields the lemma.

If $T$ is a subset of $S_{r}^{2 k}$ let $\mathscr{Q}(T)_{k}$ be the subspace of $\mathscr{Q}(r)_{k}$ generated by all $\Pi_{a=1}^{2 k} \theta\left[m_{a}\right]$ such that $\left(m_{1}, \ldots, m_{2 k}\right) \in T$. If $x=\left(m_{1}, \ldots, m_{2 k}\right)$ we use the notation

$$
\theta[x]=\prod_{a=1}^{2 k} \theta\left[m_{a}\right]=\theta\left[m_{1} \cdots m_{2 k}\right] .
$$

Lemma 6. Let $C_{1}, \ldots, C_{s}$ be the connected components of $\operatorname{Graph}\left(S_{r}^{2 k}\right)$. Then $\mathfrak{Q}(r)_{k}=\bigoplus \Sigma_{i=1}^{s} \mathbb{Q}\left(C_{i}\right)_{k}$. If $x, y \in S_{r}^{2 k}$,

$$
\sum_{a=1}^{2 k}\left(r x_{a}^{\prime}\right)^{2} \equiv \sum_{a=1}^{2 k}\left(r y_{a}^{\prime}\right)^{2} \bmod (r \operatorname{gcd}(r, 2 d, 2 e)),
$$

$d=\operatorname{gcd}\left(r x_{\alpha}^{\prime}\right), e=\operatorname{gcd}\left(r y_{\alpha}^{\prime}\right)$ then $\theta[x]$ and $\theta[y]$ are linearly independent. 
Proof. By looking at the coefficients of the Fourier expansion of $\Pi_{a=1}^{2 k} \theta\left[m_{a}\right]$ in the proof of Theorem 2 we see that if $i \neq j, f \in \mathbb{Q}\left(C_{i}\right)_{k}, g \in$ $\mathbb{Q}\left(C_{j}\right)_{k}$ then the nonvanishing of the $\lambda$ th Fourier coefficient of $f$ implies the vanishing of the $\lambda$ th Fourier coefficient of $g$. This proves the first part of the lemma. As for the second part, if the stated condition holds then Lemma 5 implies that $x$ and $y$ are not connected by an arc in $\operatorname{Graph}\left(S_{T}^{2 k}\right)$. Hence, by looking at Fourier coefficients, $\theta[x]$ and $\theta[y]$ are linearly independent. This concludes the proof.

Lemma 5 can be used to help determine the 10 connected components of $\operatorname{Graph}\left(S_{4}^{4}\right)$ :

$$
\begin{aligned}
& C_{1}=\left(\begin{array}{cccc}
0 & 0 & 0 & 0 \\
& * &
\end{array}\right),\left(\begin{array}{cccc}
1 / 2 & 1 / 2 & 1 / 2 & 1 / 2 \\
& * &
\end{array}\right) \\
& C_{2}=\left(\begin{array}{cccc}
1 / 2 & 0 & 0 & 0 \\
& * &
\end{array}\right),\left(\begin{array}{cccc}
1 / 4 & 1 / 4 & 1 / 4 & 1 / 4 \\
* & *
\end{array}\right),\left(\begin{array}{cccc}
1 / 2 & 1 / 2 & 1 / 2 & 0 \\
*
\end{array}\right) \text {, } \\
& C_{3}=\left(\begin{array}{cccc}
1 / 4 & 0 & 0 & 0 \\
* & *
\end{array}\right),\left(\begin{array}{cccc}
1 / 4 & 1 / 2 & 1 / 2 & 0 \\
*
\end{array}\right), \quad C_{4}=\left(\begin{array}{cccc}
1 / 4 & 1 / 4 & 0 & 0 \\
* & *
\end{array}\right),\left(\begin{array}{cccc}
1 / 4 & 1 / 4 & 1 / 2 & 1 / 2 \\
* &
\end{array}\right) \text {, } \\
& C_{5}=\left(\begin{array}{cccc}
1 / 2 & 1 / 2 & 0 & 0 \\
* &
\end{array}\right), \quad C_{6}=\left(\begin{array}{cccc}
1 / 4 & 1 / 2 & 1 / 2 & 1 / 2 \\
* &
\end{array}\right), \quad C_{7}=\left(\begin{array}{cccc}
1 / 4 & 1 / 2 & 0 & 0 \\
*
\end{array}\right) \text {, }
\end{aligned}
$$

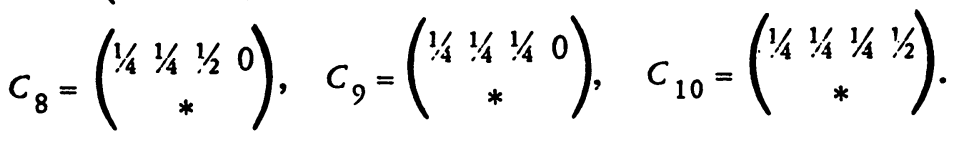

Here * refers to all 4-tuples such that $\left(\begin{array}{l}x \\ *\end{array}\right) \in S_{4^{*}}^{4}$

We now derive a finer decomposition than that given in Lemma 6 . If $L$ is a subset of $\Gamma(1)$ define an equivalence relation $*(L)$ on $S_{r}^{2 k}$ as follows: If $x$ and $y$ are in $S_{r}^{2 k}, x *(L) y$ means that, for all $M$ in $L, M x$ and $M y$ are $\sim$ to elements in the same connected component of $\operatorname{Graph}\left(S_{r}^{2 k}\right)$. (This component may change with $M_{\text {.) }}$

Lemma 7. If $L$ is a subset of $\Gamma(1)$, let $E_{1}, \ldots, E_{t}$ be the $*(L)$. equivalence classes in $S_{r}^{2 k}$. T.hen

$$
\mathfrak{Q}(r)_{k}=\bigoplus \sum_{i=1}^{t} \mathfrak{Q}\left(E_{i}\right)_{k} .
$$

Proof. Suppose $f_{1}+\cdots+f_{t}=0, f_{i}$ in $\mathbb{Q}\left(E_{i}\right)_{k}, i=1, \ldots, t$. The definition of $*(L)$ and the transformation law imply there exists $M$ in $L$ such that $\left.f_{1}\right|_{k} M^{-1}$ is in $\mathbb{Q}(S)_{k}$ and $f_{t} l_{k} M^{-1}$. is in $\mathscr{Q}(T)_{k}$ where $S$ and $T$ are 
distinct components of $\operatorname{Graph}\left(S_{r}^{2 k}\right)$. After possible rearrangement we have

$$
\left.f_{1}\right|_{k} M^{-1}+\cdots+\left.f_{q}\right|_{k} M^{-1}+\left.f_{q+1}\right|_{k} M^{-1}+\cdots+\left.f_{t}\right|_{k} M^{-1}=0
$$

where the first $q$ summands are in $\mathbb{Q}(S)_{k}$ and the last $t-q$ summands are in various $\mathbb{Q}\left(S_{i}\right)_{k}$, the $S_{i}$ being connected components distinct from $S$. By Lemma 6,

$$
\left.f_{1}\right|_{k} M^{-1}+\cdots+\left.f_{q}\right|_{k} M^{-1}=0,\left.\quad f_{q+1}\right|_{k} M^{-1}+\cdots+\left.f_{t}\right|_{k} M^{-1}=0 .
$$

Thus $f_{1}+\cdots+f_{q}=0, f_{q+1}+\cdots+f_{t}=0$. Repeating the same process to each of these equations eventually gives $f_{1}=\cdots=f_{t}=0$, thereby proving the lemma.

Theorem 4. The space of cusp forms of weight 2 (differentials of the first kind) for the principal congruence subgroup $\Gamma(8)$ has as a basis the following monomials of theta constants:

$$
\begin{gathered}
\theta\left[\begin{array}{cccc}
1 / 2 & 1 / 2 & 0 & 0 \\
1 / 4 & 1 / 4 & 1 / 4 & 1 / 4
\end{array}\right], \quad \theta\left[\begin{array}{cccc}
1 / 4 & 1 / 4 & 1 / 4 & 1 / 4 \\
1 / 2 & 1 / 2 & 0 & 0
\end{array}\right], \quad \theta\left[\begin{array}{cccc}
1 / 2 & 0 & 0 & 0 \\
1 / 4 & 1 / 4 & 1 / 4 & 1 / 4
\end{array}\right], \\
\theta\left[\begin{array}{llll}
0 & 0 & 0 & 1 / 2 \\
0 & 0 & 1 / 2 & 0
\end{array}\right], \quad \theta\left[\begin{array}{llll}
1 / 2 & 1 / 2 & 1 / 2 & 0 \\
1 / 4 & 1 / 4 & 1 / 4 & 1 / 4
\end{array}\right]
\end{gathered}
$$

Proof. These monomials are elements of $A(\Gamma(8))_{2}$ by [3, p. 235]. They are cusp forms for $\Gamma(16,32)$ by the Corollary of Theorem 2 . Since $\Gamma(16,32)$ is a normal subgroup of $\Gamma(8)$ we have $C(\Gamma(8))_{2}=C(\Gamma(16,32))_{2} \cap$ $A(\Gamma(8))_{2}$, implying the above monomials are in $C(\Gamma(8))_{2}$. Dim $C(\Gamma(8))_{2}=$ genus $(\Gamma(8))=5$. There remains to show that the monomials are linearly independent. We shall show that these monomials are in different direct summands of Lemma 7. In that lemma take $L=\left(\begin{array}{ll}1 & 0 \\ 0 & 1\end{array}\right),\left(\begin{array}{rr}0 & 1 \\ -1 & 0\end{array}\right),\left(\begin{array}{rr}0 & 1 \\ -1 & -1\end{array}\right)$, and denote these matrices by $A, B, C$ respectively. Then

$$
A\left(\begin{array}{l}
m^{\prime} \\
m^{\prime \prime}
\end{array}\right)=\left(\begin{array}{c}
m^{\prime} \\
m^{\prime \prime}
\end{array}\right), \quad B\left(\begin{array}{c}
m^{\prime} \\
m^{\prime \prime}
\end{array}\right)=\left(\begin{array}{c}
m^{\prime \prime} \\
-m^{\prime}
\end{array}\right), \quad C\left(\begin{array}{l}
m^{\prime} \\
m^{\prime \prime}
\end{array}\right)=\left(\begin{array}{c}
m^{\prime \prime}-m^{\prime}+1 / 2 \\
-m^{\prime}
\end{array}\right) .
$$

Applying $\left.\right|_{k} A^{-1}$ to the above monomials implies the first monomial is independent of the space generated by the last four. Applying $\left.\right|_{k} B^{-1}$ implies the second monomial is independent of the space generated by the last three. Applying $\mathrm{I}_{k} \mathrm{C}^{-1}$ implies the fourth is independent of the space generated by the third and fifth. Finally the third and fifth are independent by the second part of Lemma 6 , thereby concluding the proof. 
In order to derive an analogous theorem for $\Gamma(16,32)$, we will need Riemann's theta formula. Let $m_{1}, m_{2}, m_{3}, m_{4}$ be real 2-component columns

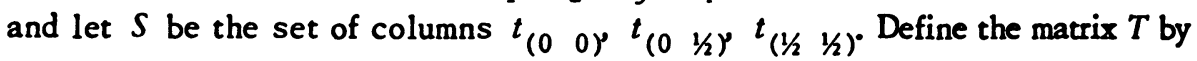

$$
T=1 / 2\left(\begin{array}{rrrr}
1 & 1 & 1 & 1 \\
1 & 1 & -1 & -1 \\
1 & -1 & 1 & -1 \\
1 & -1 & -1 & 1
\end{array}\right)
$$

and write $\left(n_{1} n_{2} n_{3} n_{4}\right)=\left(m_{1} m_{2} m_{3} m_{4}\right) T$. Reimann's theta formula is

$$
\begin{aligned}
\theta\left[m_{1}\right] \theta\left[m_{2}\right] \theta\left[m_{3}\right] \theta\left[m_{4}\right] \\
\quad=1 / 2 \sum_{a \in S} e\left(-2 m_{1}^{\prime \prime} a^{\prime \prime}\right) \theta\left[n_{1}+a\right] \theta\left[n_{2}+a\right] \theta\left[n_{3}+a\right] \theta\left[n_{4}+a\right] .
\end{aligned}
$$

The following 18 relations are all the nonredundant relations implied by the above formula which involve only monomial cusp forms in $\mathbb{Q}(4)_{2}$. The notation $x=\operatorname{LC} y, z, w$ means $x$ is a linear combination of $y, z, w$. We have also used the begining of $\$ 2$ to simplify the expressions.

$$
\begin{aligned}
& \theta\left[\begin{array}{cccc}
\frac{1}{4} & \frac{1}{4} & \frac{1}{4} & \frac{1}{4} \\
\frac{1}{2} & 0 & 0 & 0
\end{array}\right]=\text { LC } \theta\left[\begin{array}{cccc}
\frac{1}{2} & 0 & 0 & 0 \\
\frac{1}{4} & \frac{1}{4} & \frac{1}{4} & \frac{1}{4}
\end{array}\right], \quad \theta\left[\begin{array}{cccc}
0 & \frac{1}{2} & \frac{1}{2} & \frac{1}{2} \\
\frac{1}{4} & \frac{1}{4} & \frac{1}{4} & \frac{1}{4}
\end{array}\right] \text {. } \\
& \theta\left[\begin{array}{cccc}
\frac{1}{4} & \frac{1}{4} & \frac{1}{4} & \frac{1}{4} \\
\frac{1}{2} & \frac{1}{2} & \frac{1}{2} & 0
\end{array}\right]=\text { LC } \theta\left[\begin{array}{cccc}
\frac{1}{2} & 0 & 0 & 0 \\
\frac{1}{4} & \frac{1}{4} & \frac{1}{4} & \frac{1}{4}
\end{array}\right], \quad \theta\left[\begin{array}{cccc}
0 & \frac{1}{2} & \frac{1}{2} & \frac{1}{2} \\
\frac{1}{4} & \frac{1}{4} & \frac{1}{4} & \frac{1}{4}
\end{array}\right] \text {, }
\end{aligned}
$$$$
\theta\left[\begin{array}{llll}
\frac{1}{4} & \frac{1}{4} & \frac{1}{4} & \frac{1}{4} \\
\frac{1}{4} & \frac{1}{4} & 0 & 0
\end{array}\right]=\text { LC } \theta\left[\begin{array}{cccc}
\frac{1}{2} & 0 & 0 & 0 \\
\frac{1}{4} & \frac{1}{4} & 0 & 0
\end{array}\right], \quad \theta\left[\begin{array}{cccc}
\frac{1}{2} & 0 & 0 & 0 \\
\frac{1}{4} & \frac{1}{4} & \frac{1}{2} & \frac{1}{2}
\end{array}\right], \quad \theta\left[\begin{array}{cccc}
0 & \frac{1}{2} & \frac{1}{2} & \frac{1}{2} \\
\frac{1}{4} & \frac{1}{4} & 0 & 0
\end{array}\right],
$$$$
\theta\left[\begin{array}{llll}
\frac{1}{4} & \frac{1}{4} & \frac{1}{4} & \frac{1}{4} \\
\frac{1}{4} & \frac{1}{4} & \frac{1}{2} & \frac{1}{2}
\end{array}\right]=\text { LC } \theta\left[\begin{array}{llll}
\frac{1}{2} & 0 & 0 & 0 \\
\frac{1}{4} & \frac{1}{4} & 0 & 0
\end{array}\right], \quad \theta\left[\begin{array}{cccc}
\frac{1}{2} & 0 & 0 & 0 \\
\frac{1}{4} & \frac{1}{4} & \frac{1}{2} & \frac{1}{2}
\end{array}\right], \quad \theta\left[\begin{array}{cccc}
0 & \frac{1}{2} & \frac{1}{2} & \frac{1}{2} \\
\frac{1}{4} & \frac{1}{4} & 0 & 0
\end{array}\right],
$$$$
\theta\left[\begin{array}{llll}
\frac{1}{4} & \frac{1}{4} & \frac{1}{4} & \frac{1}{4} \\
\frac{3}{4} & \frac{3}{4} & 0 & 0
\end{array}\right]=\operatorname{LC} \theta\left[\begin{array}{llll}
\frac{1}{2} & 0 & 0 & 0 \\
\frac{1}{4} & \frac{1}{4} & 0 & 0
\end{array}\right], \quad \theta\left[\begin{array}{cccc}
\frac{1}{2} & 0 & 0 & 0 \\
\frac{1}{4} & \frac{1}{4} & \frac{1}{2} & \frac{1}{2}
\end{array}\right], \quad \theta\left[\begin{array}{cccc}
0 & \frac{1}{2} & \frac{1}{2} & \frac{1}{2} \\
\frac{1}{4} & \frac{1}{4} & 0 & 0
\end{array}\right],
$$ 
$\theta\left[\begin{array}{llll}\frac{1}{4} & \frac{1}{4} & \frac{1}{4} & \frac{1}{4} \\ \frac{3}{4} & \frac{3}{4} & \frac{1}{2} & \frac{1}{2}\end{array}\right]=\mathrm{LC} \theta\left[\begin{array}{cccc}\frac{1}{2} & 0 & 0 & 0 \\ \frac{1}{4} & \frac{1}{4} & 0 & 0\end{array}\right], \quad \theta\left[\begin{array}{cccc}\frac{1}{2} & 0 & 0 & 0 \\ \frac{1}{4} & \frac{1}{4} & \frac{1}{2} & \frac{1}{2}\end{array}\right], \quad \theta\left[\begin{array}{cccc}0 & \frac{1}{2} & \frac{1}{2} & \frac{1}{2} \\ \frac{1}{4} & \frac{1}{4} & 0 & 0\end{array}\right]$,

$\theta\left[\begin{array}{llll}\frac{1}{4} & \frac{1}{4} & \frac{1}{4} & \frac{1}{4} \\ \frac{1}{4} & \frac{1}{4} & \frac{1}{2} & 0\end{array}\right]=$ LC $\theta\left[\begin{array}{llll}\frac{1}{2} & 0 & 0 & 0 \\ 0 & \frac{1}{2} & \frac{1}{4} & \frac{1}{4}\end{array}\right], \quad \theta\left[\begin{array}{cccc}0 & \frac{1}{2} & \frac{1}{2} & \frac{1}{2} \\ \frac{1}{2} & 0 & \frac{1}{4} & \frac{1}{4}\end{array}\right]$

$\theta\left[\begin{array}{llll}\frac{1}{4} & \frac{1}{4} & \frac{1}{4} & \frac{1}{4} \\ \frac{1}{4} & \frac{3}{4} & \frac{1}{2} & 0\end{array}\right]=$ LC $\theta\left[\begin{array}{cccc}\frac{1}{2} & 0 & 0 & 0 \\ \frac{1}{4} & \frac{1}{4} & 0 & \frac{1}{2}\end{array}\right]$,

$\theta\left[\begin{array}{llll}\frac{1}{4} & \frac{1}{4} & \frac{1}{4} & \frac{1}{4} \\ \frac{3}{4} & \frac{3}{4} & \frac{1}{2} & 0\end{array}\right]=$ LC $\theta\left[\begin{array}{llll}\frac{1}{2} & 0 & 0 & 0 \\ 0 & \frac{1}{2} & \frac{1}{4} & \frac{1}{4}\end{array}\right], \quad \theta\left[\begin{array}{llll}0 & \frac{1}{2} & \frac{1}{2} & \frac{1}{2} \\ \frac{1}{2} & 0 & \frac{1}{4} & \frac{1}{4}\end{array}\right]$.

$\theta\left[\begin{array}{llll}\frac{1}{4} & \frac{1}{4} & 0 & 0 \\ \frac{1}{4} & \frac{1}{4} & 0 & 0\end{array}\right]=$ LC $\theta\left[\begin{array}{llll}\frac{1}{4} & \frac{1}{4} & 0 & 0 \\ \frac{3}{4} & \frac{3}{4} & \frac{1}{2} & \frac{1}{2}\end{array}\right], \quad \theta\left[\begin{array}{llll}\frac{1}{4} & \frac{1}{4} & \frac{1}{2} & \frac{1}{2} \\ \frac{3}{4} & \frac{3}{4} & 0 & 0\end{array}\right]$.

$\theta\left[\begin{array}{llll}\frac{1}{4} & \frac{1}{4} & 0 & 0 \\ 0 & 0 & \frac{1}{4} & \frac{1}{4}\end{array}\right]=$ LC $\theta\left[\begin{array}{llll}\frac{1}{4} & \frac{1}{4} & 0 & 0 \\ \frac{1}{4} & \frac{3}{4} & 0 & 0\end{array}\right], \quad \theta\left[\begin{array}{llll}\frac{1}{4} & \frac{1}{4} & 0 & 0 \\ 3 & \frac{1}{4} & \frac{1}{2} & \frac{1}{2}\end{array}\right]$.

$\theta\left[\begin{array}{llll}\frac{1}{4} & \frac{1}{4} & 0 & 0 \\ 0 & \frac{1}{4} & \frac{1}{4} & 0\end{array}\right]=$ LC $\quad \theta\left[\begin{array}{cccc}\frac{1}{4} & \frac{1}{4} & 0 & 0 \\ \frac{3}{4} & \frac{1}{2} & \frac{1}{2} & \frac{1}{4}\end{array}\right], \quad \theta\left[\begin{array}{cccc}\frac{1}{4} & \frac{1}{4} & \frac{1}{2} & \frac{1}{2} \\ \frac{3}{4} & 0 & 0 & \frac{1}{4}\end{array}\right]$.

$\theta\left[\begin{array}{llll}\frac{1}{4} & \frac{1}{4} & 0 & 0 \\ \frac{1}{2} & \frac{1}{2} & \frac{1}{4} & \frac{1}{4}\end{array}\right]=$ LC $\theta\left[\begin{array}{cccc}\frac{1}{4} & \frac{1}{4} & 0 & 0 \\ \frac{3}{4} & \frac{1}{4} & 0 & 0\end{array}\right], \quad \theta\left[\begin{array}{cccc}\frac{1}{4} & \frac{1}{4} & 0 & 0 \\ \frac{1}{4} & \frac{3}{4} & \frac{1}{2} & \frac{1}{2}\end{array}\right], \quad \theta\left[\begin{array}{cccc}\frac{1}{4} & \frac{1}{4} & \frac{1}{2} & \frac{1}{2} \\ \frac{1}{4} & \frac{3}{4} & 0 & 0\end{array}\right]$,

$\theta\left[\begin{array}{llll}\frac{1}{4} & \frac{1}{4} & \frac{1}{2} & \frac{1}{2} \\ \frac{1}{2} & \frac{1}{2} & \frac{1}{4} & \frac{1}{4}\end{array}\right]=$ LC $\theta\left[\begin{array}{llll}\frac{1}{4} & \frac{1}{4} & 0 & 0 \\ \frac{1}{4} & \frac{3}{4} & 0 & 0\end{array}\right], \quad \theta\left[\begin{array}{cccc}\frac{1}{4} & \frac{1}{4} & 0 & 0 \\ \frac{3}{4} & \frac{1}{4} & \frac{1}{2} & \frac{1}{2}\end{array}\right], \quad \theta\left[\begin{array}{cccc}\frac{1}{4} & \frac{1}{4} & \frac{1}{2} & \frac{1}{2} \\ \frac{3}{4} & \frac{1}{4} & 0 & 0\end{array}\right]$, 
$\theta\left[\begin{array}{cccc}\frac{1}{4} & \frac{1}{4} & \frac{1}{2} & \frac{1}{2} \\ 0 & 0 & \frac{1}{4} & \frac{1}{4}\end{array}\right]=$ LC $\theta\left[\begin{array}{cccc}\frac{1}{4} & \frac{1}{4} & 0 & 0 \\ \frac{3}{4} & \frac{1}{4} & 0 & 0\end{array}\right],, \quad \theta\left[\begin{array}{cccc}\frac{1}{4} & \frac{1}{4} & 0 & 0 \\ \frac{1}{4} & \frac{3}{4} & \frac{1}{2} & \frac{1}{2}\end{array}\right],, \quad \theta\left[\begin{array}{cccc}\frac{1}{4} & \frac{1}{4} & \frac{1}{2} & \frac{1}{2} \\ \frac{1}{4} & \frac{3}{4} & 0 & 0\end{array}\right]$,

$\theta\left[\begin{array}{llll}\frac{1}{4} & \frac{1}{4} & \frac{1}{2} & \frac{1}{2} \\ \frac{1}{4} & 0 & \frac{1}{4} & 0\end{array}\right]=$ LC $\theta\left[\begin{array}{cccc}\frac{1}{4} & \frac{1}{4} & 0 & 0 \\ \frac{3}{4} & 0 & \frac{1}{4} & 0\end{array}\right], \quad \theta\left[\begin{array}{cccc}\frac{1}{4} & \frac{1}{4} & 0 & 0 \\ \frac{3}{4} & \frac{1}{2} & \frac{1}{4} & \frac{1}{2}\end{array}\right]$.

$\theta\left[\begin{array}{llll}\frac{1}{2} & \frac{1}{2} & 0 & 0 \\ \frac{1}{4} & \frac{1}{4} & \frac{1}{4} & \frac{1}{4}\end{array}\right]=\operatorname{LC} \theta\left[\begin{array}{cccc}0 & 0 & \frac{1}{2} & \frac{1}{2} \\ \frac{1}{2} & 0 & 0 & 0\end{array}\right]$,

$\theta\left[\begin{array}{llll}\frac{1}{4} & \frac{1}{4} & \frac{1}{4} & \frac{1}{4} \\ \frac{1}{2} & \frac{1}{2} & 0 & 0\end{array}\right]=\operatorname{LC} \theta\left[\begin{array}{llll}\frac{1}{2} & 0 & 0 & 0 \\ 0 & 0 & \frac{1}{2} & \frac{1}{2}\end{array}\right]$.

There are many other relations implied by Riemann's formula but they fail to satisfy the conditions we imposed. For example,

$$
\theta\left[\begin{array}{cccc}
1 / 2 & 1 / 2 & 1 / 2 & 1 / 2 \\
1 / 4 & 1 / 4 & 0 & 0
\end{array}\right]=\operatorname{LC} \theta\left[\begin{array}{cccc}
0 & 0 & 0 & 0 \\
1 / 4 & 1 / 4 & 0 & 0
\end{array}\right], \theta\left[\begin{array}{cccc}
0 & 0 & 0 & 0 \\
1 / 4 & 1 / 4 & 1 / 2 & 1 / 2
\end{array}\right],
$$

involves noncusp form monomials on the right side and

$$
\theta\left[\begin{array}{llll}
\frac{1}{4} & 0 & 0 & 0 \\
\frac{1}{4} & 0 & 0 & 0
\end{array}\right]=\theta\left[\begin{array}{llll}
\frac{1}{8} & \frac{1}{8} & \frac{1}{8} & \frac{1}{8} \\
\frac{1}{8} & \frac{1}{8} & \frac{1}{8} & \frac{1}{8}
\end{array}\right]+\cdots
$$

involves characteristics with denominator 8 .

Theorem 5. The space of cusp forms of weight 2 (differentials of the first kind) for the congruence group $\Gamma(16,32)$ is contained in the space generated by monomials of theta constants $\theta\left[m_{1}\right] \theta\left[m_{2}\right] \theta\left[m_{3}\right] \theta\left[m_{4}\right], 16 m_{i} \equiv$ $0(\bmod 1), i=1, \ldots, 4$. A basis for this space of cusp forms is given by the 12 expressions in the Corollary of Theorem 3 and the set of all monomial cusp forms (determined in the Corollary of Theorem 2) not including the 18 monomials on the left side of the above Riemann theta relations.

Proof. First note that the number of elements in the proposed basis 
is $12+(423-18)=417$ as was shown at the end of $\$ 2$. Moreover $\operatorname{Dim} C(\Gamma(16,32))_{2}=417$ by [1]. There remains to show that the proposed basis elements are linearly independent. We can accomplish this with the help of the direct sum decomposition of Lemma 7, using the same $L$ as was used in the proof of Theorem 4. Since the complete listing of the $*(L)$ equivalence classes would be too lengthy to include here, we shall give an example of one such class and the analysis of it:

$$
\left(\begin{array}{llll}
1 / 4 & 1 / 4 & 1 / 4 & 1 / 4 \\
1 / 4 & 1 / 4 & 1 / 2 & 0
\end{array}\right),\left(\begin{array}{llll}
1 / 4 & 1 / 4 & 1 / 4 & 1 / 4 \\
3 / 4 & 3 / 4 & 1 / 2 & 0
\end{array}\right),\left(\begin{array}{llll}
1 / 2 & 1 / 2 & 1 / 2 & 0 \\
1 / 4 & 1 / 4 & 0 & 1 / 2
\end{array}\right),\left(\begin{array}{cccc}
1 / 2 & 0 & 0 & 0 \\
0 & 1 / 2 & 1 / 4 & 1 / 4
\end{array}\right) .
$$

Looking at the Riemann theta formula, monomials associated with the first two are in the space generated by the monomials associated with the last two. The monomials associated with the last two are linearly independent by the second part of Lemma 6 and they are cusp forms by the Corollary of Theorem 2.

\section{REFERENCES}

1. A. J. Crisalli, Theta constants and cusp forms, Trans. Amer. Math. Soc. 201 (1975), 125-132.

2. J. Igusa, On the graded ring of theta-constants, Amer. J. Math. 86 (1964), 219-246. MR 29\#2258.

3. - On the graded ring of theta-constants. II, Amer. J. Math. 88 (1966), 221-236. MR 34 \#375.

4. - Geometric and analytic methods in the theory of theta-functions, Algebraic Geometry (Internat. Colloq., Tata Inst. Fund. Res., Bombay, 1968), Oxford Univ. Press, London, 1969, pp. 241-253. MR $42 \# 6000$.

DEPARTMENT OF MATHEMATICS, PURDUE UNIVERSITY, WEST LAFAYETTE, INDIANA 47907 